\title{
On generalized double statistical convergence in a random 2-normed space
}

Ekrem Savas ${ }^{*}$

\section{"Correspondence:}

ekremsavas@yahoo.com;

esavas@iticu.edu.tr

Department of Mathematics,

Istanbul Commerce University,

Uskudar, Istanbul, Turkey

\begin{abstract}
Recently, the concept of statistical convergence has been studied in 2-normed and random 2-normed spaces by various authors. In this paper, we shall introduce the concept of $\lambda$-double statistical convergence and $\lambda$-double statistical Cauchy in a random 2-normed space. We also shall prove some new results.
\end{abstract}

MSC: 40A05; 40B50; 46A19; 46A45

Keywords: statistical convergence; $\lambda$-double statistical convergence; $t$-norm; 2-norm; random 2-normed space

\section{Introduction}

The probabilistic metric space was introduced by Menger [1] which is an interesting and an important generalization of the notion of a metric space. The theory of probabilistic normed (or metric) space was initiated and developed in [2-6]; further it was extended to random/probabilistic 2-normed spaces by Goleț [7] using the concept of 2-norm which is defined by Gähler (see [8, 9]); and Gürdal and Pehlivan [10] studied statistical convergence in 2-normed spaces. Also statistical convergence in 2-Banach spaces was studied by Gürdal and Pehlivan in [11]. Moreover, recently some new sequence spaces have been studied by Savas [12-14] by using 2-normed spaces.

In order to extend the notion of convergence of sequences, statistical convergence of sequences was introduced by Fast [15] and Schoenberg [16] independently. A lot of developments have been made in this areas after the works of S̆alát [17] and Fridy [18]. Over the years and under different names, statistical convergence has been discussed in the theory of Fourier analysis, ergodic theory and number theory. Recently, Mursaleen [19] studied $\lambda$-statistical convergence as a generalization of the statistical convergence, and in [20] he considered the concept of statistical convergence of sequences in random 2-normed spaces. Quite recently, Bipan and Savas [21] defined lacunary statistical convergence in a random 2-normed space, and also Savas [22] studied $\lambda$-statistical convergence in a random 2-normed space.

The notion of statistical convergence depends on the density of subsets of $\mathbf{N}$, the set of natural numbers. Let $K$ be a subset of $\mathbf{N}$. Then the asymptotic density of $K$ denoted by $\delta(K)$ is defined as

$$
\delta(K)=\lim _{n \rightarrow \infty} \frac{1}{n}|\{k \leq n: k \in K\}|,
$$

where the vertical bars denote the cardinality of the enclosed set.

2012 Savas; licensee Springer. This is an Open Access article distributed under the terms of the Creative Commons Attribution License (http://creativecommons.org/licenses/by/2.0), which permits unrestricted use, distribution, and reproduction in any medium, provided the original work is properly cited. 
A single sequence $x=\left(x_{k}\right)$ is said to be statistically convergent to $\ell$ if for every $\varepsilon>0$, the set $K(\varepsilon)=\left\{k \leq n:\left|x_{k}-\ell\right| \geq \varepsilon\right\}$ has asymptotic density zero, i.e.,

$$
\lim _{n \rightarrow \infty} \frac{1}{n}\left|\left\{k \leq n:\left|x_{k}-\ell\right| \geq \varepsilon\right\}\right|=0
$$

In this case we write $S-\lim x=\ell$ or $x_{k} \rightarrow \ell(S)($ see $[15,18])$.

\section{Definitions and preliminaries}

We begin by recalling some notations and definitions which will be used in this paper.

Definition 1 A function $f: \mathbf{R} \rightarrow \mathbf{R}_{0}^{+}$is called a distribution function if it is a nondecreasing and left continuous with $\inf _{t \in \mathbf{R}} f(t)=0$ and $\sup _{t \in \mathbf{R}} f(t)=1$. By $D^{+}$, we denote the set of all distribution functions such that $f(0)=0$. If $a \in \mathbf{R}_{0}^{+}$, then $H_{a} \in D^{+}$, where

$$
H_{a}(t)= \begin{cases}1, & \text { if } t>a \\ 0, & \text { if } t \leq a\end{cases}
$$

It is obvious that $H_{0} \geq f$ for all $f \in D^{+}$.

A $t$-norm is a continuous mapping $*:[0,1] \times[0,1] \rightarrow[0,1]$ such that $([0,1], *)$ is an Abelian monoid with unit one and $c * d \geq a * b$ if $c \geq a$ and $d \geq b$ for all $a, b, c, d \in[0,1]$. A triangle function $\tau$ is a binary operation on $D^{+}$, which is commutative, associative and $\tau\left(f, H_{0}\right)=f$ for every $f \in D^{+}$.

In [8], Gähler introduced the following concept of a 2-normed space.

Definition 2 Let $X$ be a real vector space of dimension $d>1$ ( $d$ may be infinite). A realvalued function $\|\cdot, \cdot\|$ from $X^{2}$ into $\mathbf{R}$ satisfying the following conditions:

(1) $\left\|x_{1}, x_{2}\right\|=0$ if and only if $x_{1}, x_{2}$ are linearly dependent,

(2) $\left\|x_{1}, x_{2}\right\|$ is invariant under permutation,

(3) $\left\|\alpha x_{1}, x_{2}\right\|=|\alpha|\left\|x_{1}, x_{2}\right\|$, for any $\alpha \in \mathbf{R}$,

(4) $\left\|x+\bar{x}, x_{2}\right\| \leq\left\|x, x_{2}\right\|+\left\|\bar{x}, x_{2}\right\|$

is called a 2-norm on $X$ and the pair $(X,\|\cdot, \cdot\|)$ is called a 2-normed space.

A trivial example of a 2-normed space is $X=\mathbf{R}^{2}$, equipped with the Euclidean 2-norm $\left\|x_{1}, x_{2}\right\|_{E}=$ the area of the parallelogram spanned by the vectors $x_{1}, x_{2}$ which may be given explicitly by the formula

$$
\left\|x_{1}, x_{2}\right\|_{E}=\left|\operatorname{det}\left(x_{i j}\right)\right|=\operatorname{abs}\left(\operatorname{det}\left(\left\langle x_{i}, x_{j}\right\rangle\right)\right),
$$

where $x_{i}=\left(x_{i 1}, x_{i 2}\right) \in \mathbf{R}^{2}$ for each $i=1,2$.

Recently, Goleț [7] used the idea of a 2-normed space to define a random 2-normed space.

Definition 3 Let $X$ be a linear space of dimension $d>1$ ( $d$ may be infinite), $\tau$ a triangle, and $\mathcal{F}: X \times X \rightarrow D^{+}$. Then $\mathcal{F}$ is called a probabilistic 2 -norm and $(X, \mathcal{F}, \tau)$ a probabilistic 2-normed space if the following conditions are satisfied: 
$\left(P 2 N_{1}\right) \mathcal{F}(x, y ; t)=H_{0}(t)$ if $x$ and $y$ are linearly dependent, where $\mathcal{F}(x, y ; t)$ denotes the value of $\mathcal{F}(x, y)$ at $t \in \mathbf{R}$,

$\left(P 2 N_{2}\right) \mathcal{F}(x, y ; t) \neq H_{0}(t)$ if $x$ and $y$ are linearly independent,

$\left(P 2 N_{3}\right) \mathcal{F}(x, y ; t)=\mathcal{F}(y, x ; t)$, for all $x, y \in X$,

$\left(P 2 N_{4}\right) \mathcal{F}(\alpha x, y ; t)=\mathcal{F}\left(x, y ; \frac{t}{|\alpha|}\right)$, for every $t>0, \alpha \neq 0$ and $x, y \in X$,

$\left(P 2 N_{5}\right) \mathcal{F}(x+y, z ; t) \geq \tau(\mathcal{F}(x, z ; t), \mathcal{F}(y, z ; t))$, whenever $x, y, z \in X$.

If $\left(P 2 N_{5}\right)$ is replaced by

$\left(P 2 N_{6}\right) \mathcal{F}\left(x+y, z ; t_{1}+t_{2}\right) \geq \mathcal{F}\left(x, z ; t_{1}\right) * \mathcal{F}\left(y, z ; t_{2}\right)$, for all $x, y, z \in X$ and $t_{1}, t_{2} \in \mathbf{R}_{0}^{+}$

then $(X, \mathcal{F}, *)$ is called a random 2-normed space (for short, R2NS).

Remark 1 Every 2-normed space $(X,\|\cdot, \cdot\|)$ can be made a random 2-normed space in a natural way by setting $\mathcal{F}(x, y ; t)=H_{0}(t-\|x, y\|)$ for every $x, y \in X, t>0$ and $a * b=\min \{a, b\}$, $a, b \in[0,1]$.

Example 1 Let $(X,\|\cdot, \cdot\|)$ be a 2-normed space with $\|x, z\|=\left\|x_{1} z_{2}-x_{2} z_{1}\right\|, x=\left(x_{1}, x_{2}\right), z=$ $\left(z_{1}, z_{2}\right)$ and $a * b=a b, a, b \in[0,1]$. For all $x \in X, t>0$ and nonzero $z \in X$, consider

$$
\mathcal{F}(x, z ; t)= \begin{cases}\frac{t}{t+\|x, z\|}, & \text { if } t>0 \\ 0, & \text { if } t \leq 0\end{cases}
$$

Then $(X, F, *)$ is a random 2-normed space.

Definition 4 A sequence $x=\left(x_{k, l}\right)$ in a random 2-normed space $(X, \mathcal{F}, *)$ is said to be double convergent (or $\mathcal{F}$-convergent) to $\ell \in X$ with respect to $\mathcal{F}$ if for each $\varepsilon>0, \eta \in(0,1)$, there exists a positive integer $n_{0}$ such that $\mathcal{F}\left(x_{k, l}-\ell, z ; \varepsilon\right)>1-\eta$, whenever $k, l \geq n_{0}$ and for nonzero $z \in X$. In this case we write $\mathcal{F}-\lim _{k, l} x_{k, l}=\ell$, and $\ell$ is called the $\mathcal{F}$-limit of $x=\left(x_{k, l}\right)$.

Definition 5 A sequence $x=\left(x_{k, l}\right)$ in a random 2-normed space $(X, \mathcal{F}, *)$ is said to be double Cauchy with respect to $\mathcal{F}$ if for each $\varepsilon>0, \eta \in(0,1)$ there exist $N=N(\varepsilon)$ and $M=M(\varepsilon)$ such that $\mathcal{F}\left(x_{k, l}-x_{p, q}, z ; \varepsilon\right)>1-\eta$, whenever $k, p \geq N$ and $l, q \geq M$ and for nonzero $z \in X$.

Definition 6 A sequence $x=\left(x_{k, l}\right)$ in a random 2-normed space $(X, \mathcal{F}, *)$ is said to be double statistically convergent or $S^{2 R 2 N}$-convergent to some $\ell \in X$ with respect to $\mathcal{F}$ if for each $\varepsilon>0, \eta \in(0,1)$ and for nonzero $z \in X$ such that

$$
\delta\left(\left\{(k, l) \in \mathbf{N} \times \mathbf{N}: \mathcal{F}\left(x_{k, l}-\ell, z ; \varepsilon\right) \leq 1-\eta\right\}\right)=0 .
$$

In other words, we can write the sequence $\left(x_{k, l}\right)$ double statistically converges to $\ell$ in random 2-normed space $(X, \mathcal{F}, *)$ if

$$
\lim _{m, n \rightarrow \infty} \frac{1}{m n}\left|\left\{k \leq m, l \leq n: \mathcal{F}\left(x_{k, l}-\ell, z ; \varepsilon\right) \leq 1-\eta\right\}\right|=0
$$


or equivalently,

$$
\delta\left(\left\{k, l \in \mathbf{N}: \mathcal{F}\left(x_{k, l}-\ell, z ; \varepsilon\right)>1-\eta\right\}\right)=1,
$$

i.e.,

$$
S^{2}-\lim _{k, l \rightarrow \infty} \mathcal{F}\left(x_{k, l}-\ell, z ; \varepsilon\right)=1
$$

In this case we write $S^{2 R 2 N}-\lim x=\ell$, and $\ell$ is called the $S^{2 R 2 N}$-limit of $x$. Let $S^{2 R 2 N}(X)$ denote the set of all double statistically convergent sequences in a random 2-normed space $(X, \mathcal{F}, *)$.

In this article, we study $\lambda$-double statistical convergence in a random 2-normed space which is a new and interesting idea. We show that some properties of $\lambda$-double statistical convergence of real numbers also hold for sequences in random 2-normed spaces. We establish some relations related to double statistically convergent and $\lambda$-double statistically convergent sequences in random 2-normed spaces.

\section{$3 \lambda$-double statistical convergence in a random 2-normed space}

Recently, the concept of $\lambda$-double statistical convergence has been introduced and studied in [23] and [24]. In this section, we define $\lambda$-double statistically convergent sequence in a random 2-normed space $(X, \mathcal{F}, *)$. Also we get some basic properties of this notion in a random 2-normed space.

Definition 7 Let $\lambda=\left(\lambda_{n}\right)$ and $\mu=\left(\mu_{n}\right)$ be two non-decreasing sequences of positive real numbers such that each is tending to $\infty$ and

$$
\lambda_{n+1} \leq \lambda_{n}+1, \quad \lambda_{1}=1
$$

and

$$
\mu_{n+1} \leq \mu_{n}+1, \quad \mu_{1}=1
$$

Let $K \subseteq \mathbb{N} \times \mathbb{N}$. The number

$$
\delta_{\bar{\lambda}}(K)=\lim _{m n} \frac{1}{\bar{\lambda}_{m n}}\left|\left\{k \in I_{n}, l \in J_{m}:(k, l) \in K\right\}\right|,
$$

where $I_{n}=\left[n-\lambda_{n}+1, n\right], J_{m}=\left[m-\mu_{m}+1, m\right]$ and $\bar{\lambda}_{n m}=\lambda_{n} \mu_{m}$, is said to be the $\lambda$-double density of $K$, provided the limit exists.

Definition 8 A sequence $x=\left(x_{k, l}\right)$ is said to be $\lambda$-double statistically convergent or $S_{\bar{\lambda}}^{2}$ convergent to the number $\ell$ if for every $\varepsilon>0$, the set $N(\varepsilon)$ has $\lambda$-double density zero, where

$$
N(\varepsilon)=\left\{k \in I_{n}, l \in J_{m}:\left|x_{k, l}-\ell\right| \geq \varepsilon\right\} .
$$

In this case, we write $S_{\bar{\lambda}}^{2}-\lim x=L$. 
Now we define $\lambda$-double statistical convergence in a random 2-normed space (see [25]).

Definition 9 A sequence $x=\left(x_{k, l}\right)$ in a random 2-normed space $(X, \mathcal{F}, *)$ is said to be $\lambda$-double statistically convergent or $S_{\bar{\lambda}}^{2}$-convergent to $\ell \in X$ with respect to $\mathcal{F}$ if for every $\varepsilon>0, \eta \in(0,1)$ and for nonzero $z \in X$ such that

$$
\delta_{\bar{\lambda}}\left(\left\{k \in I_{n}, l \in J_{m}: \mathcal{F}\left(x_{k, l}-\ell, z ; \varepsilon\right) \leq 1-\eta\right\}\right)=0
$$

or equivalently,

$$
\delta_{\bar{\lambda}}\left(\left\{k \in I_{n}, l \in J_{m}: \mathcal{F}\left(x_{k, l}-\ell, z ; \varepsilon\right)>1-\eta\right\}\right)=1,
$$

i.e.,

$$
S_{\bar{\lambda}}^{2}-\lim _{k, l \rightarrow \infty} \mathcal{F}\left(x_{k, l}-\ell, z ; \varepsilon\right)=1 .
$$

In this case we write $S_{\bar{\lambda}}^{2 R 2 N}-\lim x=\ell$ or $x_{k, l} \rightarrow \ell\left(S_{\bar{\lambda}}^{2 R 2 N}\right)$ and

$$
S_{\bar{\lambda}}^{2 R 2 N}(X)=\left\{x=\left(x_{k, l}\right): \exists \ell \in \mathbf{R}, S_{\bar{\lambda}}^{2 R 2 N}-\lim x=\ell\right\} .
$$

Let $S_{\bar{\lambda}}^{2 R 2 N}(X)$ denote the set of all $\lambda$-double statistically convergent sequences in a random 2-normed space $(X, \mathcal{F}, *)$.

If $\bar{\lambda}_{m n}=m n$ for every $n, m$ then $\lambda$-double statistically convergent sequences in a random 2-normed space $(X, \mathcal{F}, *)$ reduce to double statistically convergent sequences in a random 2-normed space $(X, \mathcal{F}, *)$.

Definition 9 immediately implies the following lemma.

Lemma 1 Let $(X, \mathcal{F}, *)$ be a random 2-normed space. If $x=\left(x_{k, l}\right)$ is a sequence in $X$, then for every $\varepsilon>0, \eta \in(0,1)$ and for nonzero $z \in X$, the following statements are equivalent:

(i) $S_{\bar{\lambda}}^{R 2 N}-\lim _{k, l \rightarrow \infty} x_{k, l}=\ell$;

(ii) $\delta_{\bar{\lambda}}\left(\left\{k \in I_{n}, l \in J_{m}: \mathcal{F}\left(x_{k, l}-\ell, z ; \varepsilon\right) \leq 1-\eta\right\}\right)=0$;

(iii) $\delta_{\bar{\lambda}}\left(\left\{k \in I_{n}, l \in J_{m}: \mathcal{F}\left(x_{k, l}-\ell, z ; \varepsilon\right)>1-\eta\right\}\right)=1$;

(iv) $S_{\bar{\lambda}}-\lim _{k, l \rightarrow \infty} \mathcal{F}\left(x_{k, l}-\ell, z ; \varepsilon\right)=1$.

Theorem 1 Let $(X, \mathcal{F}, *)$ be a random 2-normed space. If $x=\left(x_{k, l}\right)$ is a sequence in $X$ such that $S_{\bar{\lambda}}^{2 R 2 N}-\lim x_{k, l}=\ell$ exists, then it is unique.

Proof Suppose that $S_{\bar{\lambda}}^{2 R 2 N}-\lim _{k, l \rightarrow \infty} x_{k, l}=\ell_{1} ; S_{\bar{\lambda}}^{2 R 2 N}-\lim _{k, l \rightarrow \infty} x_{k, l}=\ell_{2}$, where $\left(\ell_{1} \neq \ell_{2}\right)$.

Let $\varepsilon>0$ be given. Choose $a>0$ such that $(1-a) *(1-a)>1-\varepsilon$.

Then, for any $t>0$ and for nonzero $z \in X$, we define

$$
\begin{aligned}
& K_{1}(a, t)=\left\{k \in I_{n}, l \in J_{m}: \mathcal{F}\left(x_{k, l}-\ell_{1}, z ; \frac{t}{2}\right) \leq 1-a\right\} ; \\
& K_{2}(a, t)=\left\{k \in I_{n}, l \in J_{m}: \mathcal{F}\left(x_{k, l}-\ell_{2}, z ; \frac{t}{2}\right) \leq 1-a\right\} .
\end{aligned}
$$


Since $S_{\bar{\lambda}}^{2 R 2 N}-\lim _{k, l \rightarrow \infty} x_{k, l}=\ell_{1}$ and $S_{\bar{\lambda}}^{2 R 2 N}-\lim _{k, l \rightarrow \infty} x_{k, l}=\ell_{2}$, we have Lemma 1 $\delta_{\bar{\lambda}}\left(K_{1}(a, t)\right)=0$ and $\delta_{\bar{\lambda}}\left(K_{2}(a, t)\right)=0$ for all $t>0$.

Now, let $K(a, t)=K_{1}(a, t) \cup K_{2}(a, t)$, then it is easy to observe that $\delta_{\bar{\lambda}}(K(a, t))=0$. But we have $\delta_{\bar{\lambda}}\left(K^{c}(r, t)\right)=1$.

Now, if $(k, l) \in K^{c}(a, t)$, then we have

$$
\mathcal{F}\left(\ell_{1}-\ell_{2}, z ; t\right) \geq \mathcal{F}\left(x_{k, l}-\ell_{1}, z ; \frac{t}{2}\right) * \mathcal{F}\left(x_{k, l}-\ell_{2}, z ; \frac{t}{2}\right)>(1-a) *(1-a)
$$

It follows that

$$
\mathcal{F}\left(\ell_{1}-\ell_{2}, z ; t\right)>(1-\varepsilon) \text {. }
$$

Since $\varepsilon>0$ was arbitrary, we get $\mathcal{F}\left(\ell_{1}-\ell_{2}, z ; t\right)=0$ for all $t>0$ and nonzero $z \in X$. Hence $\ell_{1}=\ell_{2}$.

This completes the proof.

Next theorem gives the algebraic characterization of $\lambda$-statistical convergence on random 2-normed spaces. We give it without proof.

Theorem 2 Let $(X, \mathcal{F}, *)$ be a random 2-normed space, and $x=\left(x_{k, l}\right)$ and $y=\left(y_{k, l}\right)$ be two sequences in $X$.

(a) If $S_{\bar{\lambda}}^{2 R 2 N}-\lim x_{k, l}=\ell$ and $c(\neq 0) \in \mathbf{R}$, then $S_{\bar{\lambda}}^{2 R 2 N}-\lim c x_{k, l}=c \ell$.

(b) If $S_{\bar{\lambda}}^{2 R 2 N}-\lim x_{k, l}=\ell_{1}$ and $S_{\bar{\lambda}}^{R 2 N}-\lim y_{k, l}=\ell_{2}$, then $S_{\bar{\lambda}}^{2 R 2 N}-\lim \left(x_{k, l}+y_{k, l}\right)=\ell_{1}+\ell_{2}$.

Theorem 3 Let $(X, \mathcal{F}, *)$ be a random 2 -normed space. If $x=\left(x_{k, l}\right)$ is a sequence in $X$ such that $\mathcal{F}-\lim x_{k, l}=\ell$, then $S_{\bar{\lambda}}^{2 R 2 N}-\lim x_{k, l}=\ell$.

Proof Let $\mathcal{F}-\lim x_{k, l}=\ell$. Then for every $\varepsilon>0, t>0$ and nonzero $z \in X$, there is a positive integer $n_{0}$ and $m_{0}$ such that

$$
\mathcal{F}\left(x_{k}-\ell, z ; t\right)>1-\varepsilon
$$

for all $k \geq n_{0}$. Since the set

$$
K(\varepsilon, t)=\left\{k \in I_{n}, l \in J_{m}: \mathcal{F}\left(x_{k, l}-\ell, z ; t\right) \leq 1-\varepsilon\right\}
$$

has at most finitely many terms. Since every finite subset of $\mathbf{N} \times \mathbf{N}$ has $\delta_{\bar{\lambda}}$-density zero, finally we have $\delta_{\bar{\lambda}}(K(\varepsilon, t))=0$. This shows that $S_{\bar{\lambda}}^{2 R 2 N}-\lim x_{k, l}=\ell$.

Remark 2 The converse of the above theorem is not true in general. It follows from the following example.

Example 2 Let $X=\mathbf{R}^{2}$, with the 2-norm $\|x, z\|=\left|x_{1} z_{2}-x_{2} z_{1}\right|, x=\left(x_{1}, x_{2}\right), z=\left(z_{1}, z_{2}\right)$ and $a * b=a b$ for all $a, b \in[0,1]$. Let $\mathcal{F}(x, y ; t)=\frac{t}{t+\|x, y\|}$, for all $x, z \in X, z_{2} \neq 0$, and $t>0$. We define a sequence $x=\left(x_{k}\right)$ by

$$
x_{k, l}= \begin{cases}(k l, 0), & \text { if } n-\left[\sqrt{\lambda_{n}}\right]+1 \leq k \leq n \text { and } m-\left[\sqrt{\mu_{m}}\right]+1 \leq k \leq m \\ (0,0), & \text { otherwise. }\end{cases}
$$


Now for every $0<\varepsilon<1$ and $t>0$, we write

$$
K_{n}(\varepsilon, t)=\left\{k \in I_{n}, l \in J_{m}: \mathcal{F}\left(x_{k, l}-\ell, z ; t\right) \leq 1-\varepsilon\right\} .
$$

Therefore, we get

$$
\delta_{\bar{\lambda}}(K(\varepsilon, t))=\lim _{n m \rightarrow \infty} \frac{\left[\sqrt{\bar{\lambda}_{n m}}\right]}{\bar{\lambda}_{n m}}=0 .
$$

This shows that $S_{\bar{\lambda}}^{2 R 2 N}-\lim x_{k, l}=0$, while it is obvious that $\mathcal{F}-\lim x_{k, l} \neq 0$.

Theorem 4 Let $(X, \mathcal{F}, *)$ be a random 2-normed space. If $x=\left(x_{k, l}\right)$ is a sequence in $X$, then $S_{\bar{\lambda}}^{2 R 2 N}-\lim x_{k, l}=\ell$ if and only if there exists a subset $K=\left\{\left(k_{n}, l_{n}\right): k_{1}<k_{2}, \ldots ; l_{1}<l_{2}, \ldots\right\} \subseteq$ $\mathbf{N} \times \mathbf{N}$ such that $\delta_{\bar{\lambda}}(K)=1$ and $\mathcal{F}-\lim _{n \rightarrow \infty} x_{k_{n}, l_{n}}=\ell$.

Proof Suppose first that $S_{\lambda}^{2 R 2 N}-\lim x_{k, l}=\ell$. Then for any $t>0, a=1,2,3, \ldots$ and nonzero $z \in X$, let

$$
A(a, t)=\left\{k \in I_{n} ; l \in J_{m}: \mathcal{F}\left(x_{k, l}-\ell, z ; t\right)>1-\frac{1}{a}\right\}
$$

and

$$
K(a, t)=\left\{k \in I_{n} ; l \in J_{m}: \mathcal{F}\left(x_{k, l}-\ell, z ; t\right) \leq 1-\frac{1}{a}\right\}
$$

Since $S_{\bar{\lambda}}^{2 R 2 N}-\lim x_{k, l}=\ell$, it follows that

$$
\delta_{\bar{\lambda}}(K(a, t))=0
$$

Now, for $t>0$ and $a=1,2,3, \ldots$, we observe that

$$
A(a, t) \supset A(a+1, t)
$$

and

$$
\delta_{\bar{\lambda}}(A(a, t))=1
$$

Now we have to show that for $(k, l) \in A(a, t), \mathcal{F}-\lim x_{k, l}=\ell$. Suppose that for some $(k, l) \in A(a, t),\left(x_{k, l}\right)$ is not convergent to $\ell$ with respect to $\mathcal{F}$. Then there exist some $s>0$ and a positive integer $k_{0}, l_{0}$ such that

$$
\left\{k \in I_{n} ; l \in J_{m}: \mathcal{F}\left(x_{k, l}-\ell, z ; t\right) \leq 1-s\right\}
$$

for all $k \geq k_{0}$ and $l \geq l_{0}$. Let

$$
A(s, t)=\left\{k \in I_{n} ; l \in J_{m}: \mathcal{F}\left(x_{k, l}-\ell, z ; t\right)>1-s\right\}
$$


for $k<k_{0}$ and $l<l_{0}$ and

$$
s>\frac{1}{a}, \quad a=1,2,3, \ldots
$$

Then we have

$$
\delta_{\bar{\lambda}}(A(s, t))=0 .
$$

Furthermore, $A(a, t) \subset A(s, t)$ implies that $\delta_{\bar{\lambda}}(A(a, t))=0$, which contradicts (3.1) as $\delta_{\bar{\lambda}}(A(a, t))=1$. Hence $\mathcal{F}-\lim x_{k, l}=\ell$.

Conversely, suppose that there exists a subset $K=\left\{\left(k_{n}, l_{n}\right): k_{1}<k_{2}, \ldots ; l_{1}<l_{2}, \ldots\right\} \subseteq$ $\mathbf{N} \times \mathbf{N}$ such that $\delta_{\bar{\lambda}}(K)=1$ and $\mathcal{F}-\lim _{n, m \rightarrow \infty} x_{k_{n}, l_{n}}=\ell$. Then for every $\varepsilon>0, t>0$ and nonzero $z \in X$, we can find a positive integer $n_{0}$ such that

$$
\mathcal{F}\left(x_{k, l}, z ; t\right)>1-\varepsilon
$$

for all $k, l \geq n_{0}$. If we take

$$
K(\varepsilon, t)=\left\{k \in I_{n} ; l \in J_{m}: \mathcal{F}\left(x_{k, l}-\ell, z ; t\right) \leq 1-\varepsilon\right\},
$$

then it is easy to see that

$$
K(\varepsilon, t) \subset \mathbf{N} \times \mathbf{N}-\left\{\left(k_{n_{0}+1}, l_{n_{0}+1}\right),\left(k_{n_{0}+2}, l_{n_{0}+2}\right), \ldots\right\},
$$

and finally,

$$
\delta_{\bar{\lambda}}(K(\varepsilon, t)) \leq 1-1=0 .
$$

Thus $S_{\bar{\lambda}}^{R 2 N}-\lim x_{k, l}=\ell$. This completes the proof.

We now have

Definition 10 A sequence $x=\left(x_{k, l}\right)$ in a random 2-normed space $(X, \mathcal{F}, *)$ is said to be $\lambda$-double statistically Cauchy with respect to $\mathcal{F}$ if for each $\varepsilon>0, \eta \in(0,1)$ and for nonzero $z \in X$, there exist $N=N(\varepsilon)$ and $M=M(\varepsilon)$ such that for all $k, m>N$ and $l, n>M$,

$$
\delta_{\bar{\lambda}}\left(\left\{k \in I_{n} ; l \in J_{m}: \mathcal{F}\left(x_{k, l}-x_{M N}, z ; \varepsilon\right) \leq 1-\eta\right\}\right)=0,
$$

or equivalently,

$$
\delta_{\bar{\lambda}}\left(\left\{k \in I_{n} ; l \in J_{m}: \mathcal{F}\left(x_{k, l}-x_{M N}, z ; \varepsilon\right)>1-\eta\right\}\right)=1 .
$$

Theorem 5 Let $(X, \mathcal{F}, *)$ be a random 2-normed space. Then a sequence $\left(x_{k, l}\right)$ in $X$ is $\lambda$ double statistically convergent if and only if it is $\lambda$-double statistically Cauchy in random 2-normed space $X$. 
Proof Let $\left(x_{k, l}\right)$ be a $\lambda$-double statistically convergent to $\ell$ with respect to random 2normed space, i.e., $S_{\bar{\lambda}}^{2 R 2 N}-\lim x_{k}=\ell$. Let $\varepsilon>0$ be given. Choose $a>0$ such that

$$
(1-a) *(1-a)>1-\varepsilon .
$$

For $t>0$ and for nonzero $z \in X$, define

$$
A(a, t)=\left\{k \in I_{n} ; l \in J_{m}: \mathcal{F}\left(x_{k, l}-\ell, z ; \frac{t}{2}\right) \leq 1-a\right\} .
$$

Then

$$
A^{c}(a, t)=\left\{k \in I_{n} ; l \in J_{m}: \mathcal{F}\left(x_{k, l}-\ell, z ; \frac{t}{2}\right)>1-a\right\} .
$$

Since $S_{\bar{\lambda}}^{2 R 2 N}-\lim x_{k, l}=\ell$, it follows that $\delta_{\bar{\lambda}}(A(a, t))=0$, and finally, $\delta_{\bar{\lambda}}\left(A^{c}(a, t)\right)=1$.

Let $p, q \in A^{c}(a, t)$. Then

$$
\mathcal{F}\left(x_{p, q}-\ell, z ; \frac{t}{2}\right)>1-a
$$

If we take

$$
B(\varepsilon, t)=\left\{k \in I_{n} ; l \in J_{m}: \mathcal{F}\left(x_{k, l}-x_{p, q}, z ; t\right) \leq 1-\varepsilon\right\},
$$

then to prove the result it is sufficient to prove that $B(\varepsilon, t) \subseteq A(a, t)$.

Let $(k, l) \in B(\varepsilon, t) \cap A^{c}(a, t)$, then for nonzero $z \in X$, we have

$$
\mathcal{F}\left(x_{k, l}-x_{p, q}, z ; t\right) \leq 1-\varepsilon \quad \text { and } \quad \mathcal{F}\left(x_{k, l}-\ell, z ; \frac{t}{2}\right)>1-a
$$

Now, from (3.1), (3.3) and (3.4), we get

$$
\begin{aligned}
1-\varepsilon & \geq \mathcal{F}\left(x_{k, l}-x_{p, q}, z ; t\right) \geq \mathcal{F}\left(x_{k, l}-\ell, z ; \frac{t}{2}\right) * \mathcal{F}\left(x_{p}-\ell, z ; \frac{t}{2}\right) \\
& >(1-a) *(1-a)>(1-\varepsilon),
\end{aligned}
$$

which is not possible. Thus $B(\varepsilon, t) \subset A(a, t)$. Since $\delta_{\bar{\lambda}}(A(a, t))=0$, it follows that $\delta_{\bar{\lambda}}(B(\varepsilon, t))=$ 0 . This shows that $\left(x_{k, l}\right)$ is $\lambda$-double statistically Cauchy.

Conversely, suppose $\left(x_{k, l}\right)$ is $\lambda$-double statistically Cauchy but not $\lambda$-double statistically convergent with respect to $\mathcal{F}$. Then for each $\varepsilon>0, t>0$ and for nonzero $z \in X$, there exist a positive integer $N=N(\varepsilon)$ and $M=M(\varepsilon)$ such that

$$
A(\varepsilon, t)=\left\{k \in I_{n} ; l \in J_{m}: \mathcal{F}\left(x_{k, l}-x_{N M}, z ; t\right) \leq 1-\varepsilon\right\} .
$$

Then

$$
\delta_{\bar{\lambda}}(A(\varepsilon, t))=0
$$


and

$$
\delta_{\bar{\lambda}}\left(A^{c}(\varepsilon, t)\right)=1 .
$$

For $t>0$, choose $a>0$ such that

$$
(1-a) *(1-a)>1-\varepsilon
$$

is satisfied, and we take

$$
B(a, t)=\left\{k \in I_{n} ; l \in J_{m}: \mathcal{F}\left(x_{k, l}-\ell, z ; \frac{t}{2}\right)>1-a\right\} .
$$

If $N, M \in B(a, t)$, then $\mathcal{F}\left(x_{N, M}-\ell, z ; \frac{t}{2}\right)>1-a$.

Since

$$
\mathcal{F}\left(x_{k, l}-x_{N M}, z ; t\right) \geq \mathcal{F}\left(x_{k, l}-\ell, z ; \frac{t}{2}\right) * \mathcal{F}\left(x_{N, M}-\ell, z ; \frac{t}{2}\right)>(1-a) *(1-a)>1-\varepsilon
$$

then we have

$$
\delta_{\bar{\lambda}}\left(\left\{x_{k, l}: \mathcal{F}\left(x_{k, l}-x_{N M}, z ; t\right)>1-\varepsilon\right\}\right)=0,
$$

i.e., $\delta_{\bar{\lambda}}\left(A^{c}(\varepsilon, t)\right)=0$, which contradicts $(3.5)$ as $\delta_{\bar{\lambda}}\left(A^{c}(\varepsilon, t)\right)=1$. Hence $\left(x_{k, l}\right)$ is $\lambda$-double statistically convergent.

This completes the proof.

\section{Competing interests}

The author declares that they have no competing interests.

Received: 12 March 2012 Accepted: 22 August 2012 Published: 25 September 2012

\section{References}

1. Menger, K: Statistical metrics. Proc. Natl. Acad. Sci. USA 28, 535-537 (1942)

2. Alsina, C, Schweizer, B, Sklar, A: Continuity properties of probabilistic norms. J. Math. Anal. Appl. 208, 446-452 (1997)

3. Schweizer, B, Sklar, A: Statistical metric spaces. Pac. J. Math. 10, 313-334 (1960)

4. Schweizer, B, Sklar, A: Probabilistic Metric Spaces. North Holland, Amsterdam (1983)

5. Sempi, C: A short and partial history of probabilistic normed spaces. Mediterr. J. Math. 3, 283-300 (2006)

6. Šerstnev, AN: On the notion of a random normed space. Dokl. Akad. Nauk SSSR 149, 280-283 (1963)

7. Goleț, I: On probabilistic 2-normed spaces. Novi Sad J. Math. 35, 95-102 (2006)

8. Gähler, S: 2-metrische Raume and ihre topologische Struktur. Math. Nachr. 26, 115-148 (1963)

9. Gähler, S: Linear 2-normietre Raume. Math. Nachr. 28, 1-43 (1965)

10. Gürdal, M, Pehlivan, S: The statistical convergence in 2-Banach spaces. Thai J. Math. 2(1), 107-113 (2004)

11. Gürdal, M, Pehlivan, S: Statistical convergence in 2-normed spaces. Southeast Asian Bull. Math. 33, $257-264$ (2009)

12. Savas, E: $\Delta^{m}$-strongly summable sequence spaces in 2-normed spaces defined by ideal convergence and an Orlicz function. Appl. Math. Comput. 217, 271-276 (2010)

13. Savas, E: On some new sequence spaces in 2-normed spaces using Ideal convergence and an Orlicz function. J. Inequal. Appl. 2010, Article Number 482392 (2010). doi:10.1155/2010/482392

14. Savas, E: A-sequence spaces in 2-normed space defined by ideal convergence and an Orlicz function. Abstr. Appl. Anal. 2011, Article ID 741382 (2011)

15. Fast, H: Sur la convergence statistique. Colloq. Math. 2, 241-244 (1951)

16. Schoenberg, IJ: The integrability of certain functions and related summability methods. Am. Math. Mon. 66, 361-375 (1959)

17. Salát, T: On statistical convergence of real numbers. Math. Slovaca 30, 139-150 (1980)

18. Fridy, JA: On statistical convergence. Analysis 5, 301-313 (1985)

19. Mursaleen, M: $\lambda$-Statistical convergence. Math. Slovaca 50,111-115 (2000)

20. Mursaleen, M: Statistical convergence in random 2-normed spaces. Acta Sci. Math. 76(1-2), 101-109 (2010) 
21. Bipan, $\mathrm{H}$, Savas, E: Lacunary statistical convergence in random 2-normed space. Preprint

22. Savaş, $\mathrm{E}: \boldsymbol{\lambda}$-statistical convergence in random 2-normed space. Iranian Journal of Science and Technology. Preprint

23. Savas, E: $\bar{\lambda}$-double sequence spaces of fuzzy real numbers defined by Orlicz function. Math. Commun. 14, 287-297 (2009)

24. Savas, E: On $\bar{\lambda}$-statistically convergent double sequences of fuzzy numbers. J. Inequal. Appl. 2008, Art. ID 147827 (2008)

25. Savas, E, Mohiuddine, SA: $\bar{\lambda}$-statistically convergent double sequences in probabilistic normed space. Math. Slovaca 62(1), 99-108 (2012)

doi:10.1186/1029-242X-2012-209

Cite this article as: Savas: On generalized double statistical convergence in a random 2-normed space. Journal of Inequalities and Applications 2012 2012:209.

Submit your manuscript to a SpringerOpen ${ }^{\circ}$ journal and benefit from:

- Convenient online submission

Rigorous peer review

Immediate publication on acceptance

- Open access: articles freely available online

- High visibility within the field

- Retaining the copyright to your article

Submit your next manuscript at $>$ springeropen.com 